\title{
Emerging Applications of the Resampling Methods in Actuarial Models
}

\author{
Krzysztof M. Ostaszewski and Grzegorz A. Rempala *
}

Uncertainty of insurance liabilities has always been the key issue in actuarial theory and practice. This is represented for instance by study and modeling of mortality in life insurance and loss distributions in traditional actuarial science. These models have evolved from early simple deterministic calculations to more sophisticated, probabilistic ones. Such probabilistic models have been traditionally built around parameters characterizing certain probability laws, e.g., Gompertz's model of force of mortality, or parametric models of the yield curve process. In this article we describe the methodology of the bootstrap, and more generally, resampling and show some of its possible advantages in describing the underlying probability distributions. We provide two detailed examples of application of the resampling methods. First, we show how bootstrap can be used successfully to enhance a parametric mortality law suggested by Carriere (1992). Next, we develop a whole company asset-liability model to study the nonparametric bootstrap alternative to lognormal and stable Paretian models of interest rate process proposed by Klein (1993). Our results indicate that bootstrap can be instrumental in understanding the rich structure of random variables on the asset and liability sides of an insurance firm balance sheet, and in error estimation in both parametric and non-parametric setting.

${ }^{*}$ The authors graciously acknowledge support from the Actuarial Education and Research Fund 


\section{Introduction}

Actuarial science has always been concerned with uncertainty and the process of modeling it. In fact, the uncertainty of insurance liabilities may be considered the very reason for the creation of actuarial science. This is clearly represented in a study of mortality and other decrements in life, disability, health insurance, and loss distribution in property/casualty insurance. The models used in such studies began as tabular ones, and then developed into a study of probability distributions, traditionally characterized by some numerical parameters. Examples of such probability models include Makeham and Gompertz laws of mortality, or the use of gamma distribution family in modeling losses, as well as the omnipresent use of normal approximations (including the lognormal distribution modeling of interest rates).

Over the last decade a score of new methodologies for statistical inference both in parametric and non-parametric setting have been based on the concept of the bootstrap, also knows under a somewhat broader term of resampling. In this work, we present the basic ideas of the bootstrap/resampling, and point out to its promising applications in actuarial modeling.

The chapter is organized as follows. In the reminder of this section we give a brief overview of the basic ideas of the bootstrap methodology in the context of parametric and non-parametric estimation as well as time series-based inferences. The subsequent two sections of the paper are devoted to the somewhat detailed discussion of two particular examples, arising from the actuarial modeling problems. In Section 2 we look at mortality estimation, studying a mortality law introduced by Carriere (1992), generalizing on the classical Gompertz mortality law. In Section 3, we proceed to a whole company asset-liability model, comparing cash flow testing results for an annuity company under two parametric assumptions of interest 
rate process and the method based on the nonparametric, bootstrapbased approach to analyzing time series data. Section 4 offers some concluding remarks.

\subsection{The Concept}

The concept of the bootstrap was first introduced in the seminal piece of Efron (1979) as an attempt to give some new perspective to an old and established statistical procedure known as jackknifing. Unlike jackknifing which is mostly concerned with calculating standard errors of statistics of interest, Efron's bootstrap achieves more ambitious goal of estimating not only the standard error but also the distribution of a statistic. In his paper Efron has considered two types of bootstrap procedures useful, respectively, for nonparametric and parametric inference. The nonparametric bootstrap relies on the consideration of the discrete empirical distribution generated by a random sample of size $n$ from an unknown distribution $F$. This empirical distribution $\widehat{F}_{n}$ assigns equal probability to each sample item. In the parametric bootstrap setting, we consider $F$ to be a member

of some prescribed parametric family and obtain $\widehat{F}_{n}$ by estimating the family parameter(s) from the data. In each case, by generating an independent identically distributed (iid) random sequence, called $a$ resample or pseudo-sequence, from the distribution $\widehat{F}_{n}$, or its appropriately smoothed version, we can arrive at new estimates of various parameters or nonparametric characteristics of the original distribution $F$. This simple idea is at root of the bootstrap methodology. The conditions for consistency of the bootstrap method were formulated in the paper of Bickel and Freedman (1981). This resulted in further extensions of the Efron's methodology to a broad range of standard applications, including quantile processes, multiple regression and stratified sampling. Singh (1981) made a further point that the bootstrap estimator of the sampling distribution of a given statistic may be more accurate than the traditional normal approximation. In fact, it turns out that for many commonly used statistics the use of the 
bootstrap is asymptotically equivalent to the use of one-term Edgeworth correction, usually with the same convergence rate, which is faster than the normal approximation.

In many recent statistical texts, the bootstrap is recommended for estimating sampling distributions, finding standard errors, and confidence sets. Although the bootstrap methods can be applied to both parametric and non-parametric models, most of the published research in the area has been concerned with the non-parametric case since that is where the most immediate practical gains might be expected. Bootstrap as well as some other resampling procedures procedures are available in recent editions of some popular statistical software packages, including SAS and S-Plus. Readers interested in gaining some background in resampling are referred to Efron and Tibishirani (1993). For a more mathematically advanced treatment of the subject, we recommend the monograph by Shao and Tu (1995) which also contains a chapter on bootstrapping time series and other dependent data sets.

\subsection{Bootstrap Standard Error and Bias Estimates}

Arguably, one of the most important practical applications of the bootstrap is in providing conceptually simple estimates of the standard error and bias for a statistic of interest. Let $\hat{\theta}_{n}$ be a statistic based on the observed sample, arriving from some unknown distribution function $F$. Assume that $\hat{\theta}_{n}$ is to estimate some (real-valued) parameter of interest $\theta$, and let us denote its standard error and bias by $\operatorname{se}_{F}\left(\hat{\theta}_{n}\right)$ and $\operatorname{bias}_{F}\left(\hat{\theta}_{n}\right)$. Since the form of the statistic $\hat{\theta}_{n}$ may be vary complicated the exact formulas for the corresponding bootstrap estimates of standard error $(B E S E)$ and bias $(B E B)$ may be quite difficult to derive. Therefore, one usually approximates both these quantities with the help of the multiple resamples. The approximation to the bootstrap estimate of standard error of $\hat{\theta}_{n}$ suggested by 
Efron (1979) is given by

$$
\widehat{s e}_{B}=\left\{\sum_{b=1}^{B}\left[\hat{\theta}^{*}(b)-\hat{\theta}_{n}^{*}(\cdot)\right]^{2} /(B-1)\right\}^{1 / 2}
$$

where $\hat{\theta}_{n}^{*}(b)$ is the original statistic $\hat{\theta}_{n}$ calculated from the $b$-th resample $(b=1, \ldots, B), \hat{\theta}_{n}^{*}(\cdot)=\sum_{b=1}^{B} \hat{\theta}_{n}^{*}(b) / B$, and $B$ is the total number of resamples (each of size $n$ ) collected with replacement from the empirical estimate of $F$ (in either parametric or non-parametric setting), By the law of large numbers

$$
\lim _{B \rightarrow \infty} \widehat{s e}_{B}=B E S E\left(\hat{\theta}_{n}\right),
$$

and for sufficiently large $n$ we expect

$$
\operatorname{BESE}\left(\hat{\theta}_{n}\right) \approx \operatorname{se}_{F}\left(\hat{\theta}_{n}\right)
$$

Similarly, for $B E B$ one can use its approximation $\widehat{\operatorname{bias}}_{B}$ based on $B$ resamples

$$
\widehat{\operatorname{bias}}_{B}=\sum_{b=1}^{B} \hat{\theta}_{n}^{*}(b) / B-\hat{\theta}_{n}
$$

Let us note that $B$, the total number of resamples, may be taken as large as we wish. For instance, it has been shown that for estimating $B E S E, B$ equal to about 250 typically gives already a satisfactory approximation, whereas for $B E B$ this number may have to be significantly increased in order to reach the desired accuracy (see Efron and Tibishirani 1993 for a discussion of these issues). Let us also note that $B E S E$ and $B E B$ can be applied to the dependent data after some modifications of the resampling procedure (see Section 1.4 below). 


\subsection{Bootstrap Confidence Intervals}

Let us now turn into the problem of using the bootstrap methodology to construct confidence intervals. This area has been a major focus of theoretical work on the bootstrap and in fact the procedure described below is not the most efficient one and can be significantly improved in both rate of convergence and accuracy. It is, however, intuitively appealing and easy to justify and seems to be working well enough for the cases considered here. For complete review of available approaches to bootstrap confidence intervals for iid data, see Efron and Tibishirani (1992). As in the case of standard error and bias estimates the methodology for bootstrap interval estimation applies also to time dependent observations as long as we modify our resampling procedure to either block or circular bootstrap sampling (see Section 1.4 below).

Let us consider $\hat{\theta}_{n}^{*}$, a bootstrap estimate of $\theta$ based on a resample of size $n$ (or, in the case of dependent observations, on $k$ blocks of length $l$, see Section 1.4 below) from the original data sequence $X_{1}, \ldots, X_{n}$, and let $G_{*}$ be its distribution function given the observed series values

$$
G_{*}(x)=\operatorname{Prob}\left\{\hat{\theta}_{n}^{*} \leq x \mid X_{1}=x_{1}, \ldots, X_{n}=x_{n}\right\} .
$$

Recall that for any distribution function $F$ and $\tau \in(0,1)$ we define the $\tau$-th quantile of $F$ (sometimes also called $\tau$-th percentile) as $F^{-1}(\tau)=\inf \{x: F(x) \geq \tau\}$. The bootstrap percentiles method gives $G_{*}^{-1}(\alpha)$ and $G_{*}^{-1}(1-\alpha)$ as, respectively, lower and upper bounds for $1-2 \alpha$ confidence interval for $\hat{\theta}_{n}$. Let us note that for most statistics $\hat{\theta}_{n}$ the form of a distribution function of the bootstrap estimator $\hat{\theta}_{n}^{*}$ is not available. In practice, $G_{*}^{-1}(\alpha)$ and $G_{*}^{-1}(1-\alpha)$ are approximated by generating $B$ pseudo-sequences $\left(X_{1}^{*}, \ldots, X_{n}^{*}\right)$, calculating the corresponding values of $\hat{\theta}_{n}^{*}(b)$ for $b=1, \ldots, B$, and then finding the empirical percentiles. In this case the number of resamples $B$ usually needs to be quite large $(B \geq 1000)$. 


\subsection{Dependent Data}

The Efron's basic bootstrap procedure fails when the observed sample points are not independent. The extension of the bootstrap method to the case of dependent data was first considered by Künsch (1989) who suggested a moving block bootstrap procedure, which takes into account the dependence structure of the data by resampling blocks of adjacent observations rather than individual data points. The method was shown to work reasonably well. Its main drawback is related to the fact that for fixed block and sample sizes, observations in the middle part of the series had typically a greater chance of being selected into a resample than the observations close to the ends. This happens because the first or last block are often shorter. To remedy this deficiency Politis and Romano (1992) suggested a method based on circular blocks, wrapping the observed time series values around the circle and then generating consecutive blocks of bootstrap data from the circle. In the case of the sample mean this method, known as a circular bootstrap, again was shown to accomplish the Edgeworth correction for dependent data.

Let $X_{1}, \ldots, X_{n}$ be a (strictly) stationary time series, and as before let $\hat{\theta}_{n}=\hat{\theta}_{n}\left(X_{1}, \ldots, X_{n}\right)$ denote a real-valued statistic estimating some unknown parameter interest $\theta$. Given the observations $X_{1}, \ldots, X_{n}$ and an integer $l(1 \leq l \leq n)$ we form the $l$ blocks $B_{t}=\left(X_{t}, \ldots, X_{t+l-1}\right)$ for $t=1, \ldots, n-l+1$. In moving blocks procedure of Künsch (1989) the bootstrap data (pseudo-time series) $\left(X_{1}^{*}, \ldots, X_{n}^{*}\right)$ is obtained by generating the resample of $k$ blocks $(k=[n / l])$ and then considering all individual (inside-block) pseudo-values of the resulting sequence $B_{1}^{*} \ldots, B_{k}^{*}$.

The circular bootstrap approach is somewhat similar except that here we generate a subsample $\tilde{B}_{1}{ }^{*} \ldots, \tilde{B}_{k}{ }^{*}$ from the $l$-blocks $\tilde{B}_{t}=\left(\tilde{X}_{t}, \ldots, \tilde{X}_{t+l-1}\right)$ for $t=1, \ldots, n$ where 


$$
\tilde{X}_{t}= \begin{cases}X_{t} & \text { if } t=1, \ldots, n \\ X_{t-n} & t=n+1, \ldots, n+l-1\end{cases}
$$

In either case, the bootstrap version of $\hat{\theta}_{n}$ is $\hat{\theta}_{n}^{*}=\hat{\theta}_{n}\left(X_{1}^{*}, \ldots, X_{n}^{*}\right)$.

The idea behind both the block and circular bootstrap is simple: by resampling blocks rather than original observations we preserve the original short-term dependence structure between the observations (although not necessarily the long-term one). For the data series for which the long term dependence is asymptotically negligible in some sense (e.g., $\alpha$-mixing sequences with appropriately fast mixing rate) the method will produce consistent estimators of the sampling distribution of $\hat{\theta}_{n}-\theta$ as long as $l, n \rightarrow \infty$ at the appropriate rate (see e.g., Shao and Tu (1995) Chapter 9, for the discussion of the appropriate assumptions). In particular, it follows that the formulas for the approximate $B E S E$ and $B E B$ given by (1) and (2), respectively as well as the method of percentiles for confidence estimation are still valid with the block and circular bootstrap. Let us also note that for $l=1$ both methods reduce to the Efron's bootstrap procedure for iid random variables.

In the next two sections we demonstrate the use of the above bootstrap techniques in both parametric and non-parametric setting.

\section{Modeling US Mortality Tables}

In this section we consider an application of the bootstrap techniques to estimating the variability of the parameters of a general mortality law proposed by Carriere (1992). We first describe the mortality model. 


\subsection{Carriere Mortality Law}

The research into a law of mortality has had a long history going back the simplest formula of De Moivre. Probably the most popular parametric model of mortality (proposed by Benjamin Gompertz) has been the one that models the force of mortality

$$
\mu_{x}=B c^{x}
$$

as an exponential function. Whereas Gompertz's model typically fits observed mortality rates fairly well at the adult ages it exhibits some deficiency in modeling the early ages. This is mostly due to the fact that in many populations the exponential growth in mortality at adult ages is preceded by a sharp mortality fall in early childhood and the hump at about age 23 (cf. e.g., Tenenbein and Vanderhoof 1980). This early years behavior of the mortality force is not easily modeled with the formula (4). There has been many attempts in the actuarial literature to modify Gompertz's law in order to obtain a better approximation for the early mortality patterns. It has been observed in Heligman and Pollard (1980) that by adding additional components to Gompertz model one can obtain a mortality law that fits early mortality patterns much better. This idea has been further extended by Carriere 1992 [referred to in the sequel as CR] who has suggested using a mixture of the extreme-value distributions: Gompertz, Inverse Gompertz, Weibull and Inverse Weibull, to model the mortality curve of a population. He suggested a mortality law based on the survival function $s(x)$ given as the mixture

$$
s(x)=\sum_{i=1}^{4} \psi_{i} s_{i}(x) .
$$

Here $\psi_{i} \geq 0$ for $i=1 \ldots, 4$, are the mixture coefficients satisfying $\sum_{i=1}^{4} \psi_{i}=1$ and $s_{i}(x)$ for $i=1, \ldots 4$ are the survival functions based on the Gompertz, Inverse Gompertz, Weibull, and Inverse Weibull distribution functions, respectively, with the corresponding 
location and scale parameters $m_{i}$ and $\sigma_{i}$. More precisely,

$$
\begin{aligned}
& s_{1}(x)=\exp \left(e^{-m_{1} / \sigma_{1}}-e^{\left(x-m_{1}\right) / \sigma_{1}}\right) \\
& s_{2}(x)=\frac{1-\exp \left(-e^{-\left(x-m_{2}\right) / \sigma_{2}}\right)}{1-\exp \left(-e_{2}^{m} / \sigma_{2}\right)} \\
& s_{3}(x)=\exp \left(-\left(x / m_{3}\right)^{m_{3} / \sigma_{3}}\right) \\
& s_{4}(x)=1-\exp \left(-\left(x / m_{4}\right)^{-m_{4} / \sigma_{4}}\right)
\end{aligned}
$$

In Carriere mortality model the Gompertz (6a) and Inverse Gompertz (6b) curves, both derived from (4), are used to model the mortality of adulthood, whereas the Weibull and the Inverse Weibull curves are used to model the mortality of childhood and early adolescence. The introduction of the last two survival functions allows fitting correctly the early years decrease in mortality as well as the hump around age 20, which are both present in many populations mortality patterns. As it has been demonstrated empirically in CR the model (5) fits quite well the patterns of mortality for the entire US population as well as, separately, US male and female mortality laws. Due to the interpretation of the survival components of the curve (5) Carriere model, unlike that of Heligman and Pollard (1980), gives also the estimates of childhood, adolescence and adulthood mortality by means

of the mixture coefficients $\psi_{i}$. In the next section we discuss a way of arriving at these estimates.

\subsection{Fitting the Mortality Curve}

We will illustrate the use of bootstrap by fitting the general mortality model (5) to the Aggregate 1990 US Male Lives Mortality Table (US Health Dept. 1990). This particular table is of special actuarial interest because it was a data source in developing 1994 GAM and 1994 UP tables. In order to fit the model (5) to the observed mortality pattern, some fitting criterion (a loss function) is needed. In his paper $\mathrm{CR}$, Carriere has considered several different loss functions but here, 
for simplicity, we will restrict ourselves to the one given by

$$
\sum_{x=0}^{100}\left(1-\frac{\hat{q}_{x}}{q_{x}}\right)^{2}
$$

where $q_{x}$ is a probability of death within a year for a life aged $x$, as obtained from the US table and $\hat{q}_{x}$ is a corresponding probability calculated using the survival function (5). The ages above $x=100$ were not included in (7) since, similarly to CR, we have found that the mortality pattern for the late ages in the table had been based on Medicare data and hence might be not representative to the entire US male population. The parameters of the survival functions (6) as well as the corresponding mixing coefficients $\psi_{i}$ 's were calculated by minimizing the loss function (7). The calculations were performed with a help of the SOLVER add-on function in the Microsoft Office 97 Excel software. The estimated values of the parameters $m_{i}, \sigma_{i}^{2}$, and $\psi_{i}$ for $i=1, \ldots 4$ are given in the Table 1 .

\begin{tabular}{c|ccc}
\hline Comp. & Location par $\left(m_{i}\right)$ & Scale par $\left(\sigma_{i}\right)$ & Mixture par $\left(\psi_{i}\right)$ \\
\hline \hline$s_{1}(\cdot)$ & 80.73 & 11.26 & 0.944 \\
$s_{2}(\cdot)$ & 42.60 & 14.44 & 0.020 \\
$s_{3}(\cdot)$ & 0.30 & 1.30 & 0.013 \\
$s_{4}(\cdot)$ & 23.65 & 7.91 & 0.022 \\
\hline
\end{tabular}

Table 1. Estimates of the Carriere survival model parameters for 1990 US Male Mortality Tables. $\psi_{4}=1-\sum_{i=1}^{3} \psi_{i}$

As we can see from the table the modes $m_{i}$ of the survival components in the Gompertz and the Inverse Gompertz case are equal to 80.73 and 42.60 respectively, whereas in the Weibull and the Inverse Weibull case they are equal to 0.3 and 23.65 , respectively. This indicated that, as intended, both Gompertz components model the later age mortality, whereas both Weibull components model the mortality of childhood and adolescence. Moreover, since $\psi_{1}=0.94$ it follows that most of the deaths in the considered population are due to the Gompertz component. A plot of the fitted curve of $\ln \left(\hat{q}_{x}\right)$ using the 
estimated values of the parameters given in Table 1 along with the values of $\ln \left(q_{x}\right)$ for $x=0, \ldots, 100$ is presented in Figure 1.

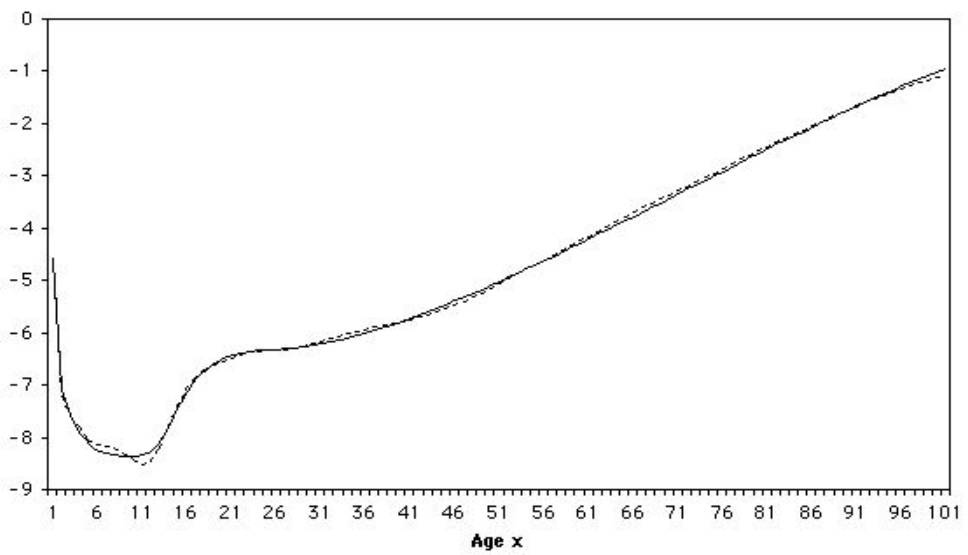

Figure 1. A plot of $\ln \left(q_{x}\right)$ from US Life Table (dashed line) and of $\ln \left(\hat{q}_{x}\right)$ using formulas (6) with the parameters values given in Table 1.

As we can see from the plot the fit seems to quite good except perhaps between the ages of 6 and 12. Let us also note the resemblance of our plot in Figure 1 to that presented in Figure 4 of CR, which was based on the 1980 US Population Mortality Table.

\subsection{Statistical Properties of the Parameter Estimates in Carriere Mortality Model}

The statistical properties of estimators of the unknown parameters $\psi_{i} i=1 \ldots 3$ and $m_{i}, \sigma_{i} i=1 \ldots, 4$ obtained by minimizing the function (7) (we refer to them in the sequel as the Carriere or $C R$ estimators) are not immediately obvious. For instance, it is not clear whether or not they are consistent and what is their asymptotic efficiency. Since these questions are related to the problem of consistency of the bootstrap estimation, we will discuss them briefly here.

In order to put our discussion in the context of the statistical infer- 
ence theory we need to note an important difference between the loss function (7) and the usual, weighted least-square loss. Namely, in our setting the US mortality rates $q_{x}$ for $x=1, \ldots, 100$ are not independent observations but rather are themselves estimated from the large number $n$ of the deaths observed in the population. ${ }^{1}$ Since the $q_{x}$ 's are estimated based on the same number $n$ of the observed deaths, they are negatively correlated and hence the loss function (7) treated as a random function, is not a sum of independent identically distributed random variables. In view of the above, the usual statistical methodology of $M$-estimation does not apply, and in order to obtain asymptotics for the $C R$ estimators we need to develop a different approach which is briefly outlined below. For further references and a general discussion of the method, see the forthcoming paper of Rempala and Szatzschneider (2002).

Under the assumption that the true mortality curve of the population indeed follows the model (5) with some unknown parameters $\psi_{i}^{0}$, $(i=1, \ldots, 3)$ and $m_{i}^{0}, \sigma_{i}^{0}(i=1, \ldots 4)$ one may consider $C R$ estimators associated with the $i$-th survival component $(i=1, \ldots, 4)$ as functions of the vector of mortality rates $q_{x}$ for $x=1, \ldots, 100$ and expand them around the true parameters $\psi_{i}^{0}, m_{i}^{0}, \sigma_{i}^{0}$ using the multivariate Taylor's Theorem. The expansion is valid in view of the Implicit Function Theorem which guarantees that all the local minimizers of (7) are locally continuously differentiable functions of the $q_{x}$ 's as long as the first derivatives of $\hat{q}_{x}=\hat{q}_{x}\left(m_{i}, \sigma_{i}, \psi_{i}\right)$ are locally continuous and do not vanish (that is, the appropriate Jacobian function is no equal to zero, at least for one age $x$ ) around the point $\psi_{i}^{0}$, $m_{i}^{0}, \sigma_{i}^{0}$. This method reveals that $C R$ estimates are consistent and that their join distribution is asymptotically multivariate normal with some covariance matrix with entries depending upon the values of the true parameters $\psi_{i}^{0},(i=1, \ldots, 3), m_{i}^{0}, \sigma_{i}^{0}(i=1, \ldots 4)$ and two first derivatives of the $\hat{q}_{x}$ 's with respect to $\psi_{i}, m_{i}, \sigma_{i}$.

\footnotetext{
${ }^{1}$ The US table used as an example in this section has, in fact, the values $q_{x}$ 's
} obtained by interpolation with the help of Beer's 4-th degree oscillatory formula. 


\subsection{Assessment of the Model Accuracy with Parametric Bootstrap}

One way of assessing the variability of $C R$ estimates would be to use the normal approximation outlined in the previous section. However, since the asymptotic variance of this approximation depends upon the unknown parameters which would have to be estimated from the data, the derivation of the exact formula for the covariance matrix as well as the assessment of the performance of variance estimators would be required. In addition, the normal approximation would not account for the possible skewness of the marginal distributions of $\psi_{i}$, $m_{i}$, and $\sigma_{i}$. Therefore, as an alternative to this traditional approach, we propose to apply the bootstrap method. Since the bootstrap typically corrects for the skewness effect we would hope that it might provide better approximations.

Let us note that the methods of the nonparametric bootstrap cannot be applied here since we do not know the empirical distribution $\widehat{F}_{n}$ of the observed deaths. However, we do know the estimated values of the parameters in the model (5), since these are obtained by minimizing the expression (7) which is concerned only with the quantities $q_{x}$. Hence, assuming that the true population death distribution follows the model (5) we may use the values given in Table 1 to obtain our resampling distribution $\widehat{F}_{n}$.

Once we have identified the resampling distribution, the bootstrap algorithm for obtaining approximate variances, biases, and confidence intervals for $C R$ estimators is quite simple. We use a computer to generate a large (in our example 20,000 points) random sample from $\widehat{F}_{n}$ and apply interpolation ${ }^{2}$ to obtain the sequence of

\footnotetext{
${ }^{2}$ For the sake of efficiency one should use here the same method of interpolation as the one used to create the original values of the $q_{x}$ 's in the table. However, in our example we simply applied linear interpolation to obtain the values of the pseudo-empirical distribution at the integer ages and then to calculate the $q_{x}^{*}$ 's
} 
the pseudo mortality rates $q_{x}^{*}$ for $x=1, \ldots, 100$. In order to find the corresponding bootstrap replication $\psi_{i}^{*} i=1 \ldots 3$ and $m_{i}^{*}, \sigma_{i}^{*}$ $i=1 \ldots, 4$ we minimize the loss function (7), where now the $q_{x}$ 's are replaced by the $q_{x}^{*}$ 's. The above procedure is repeated a large number $(B)$ times and the resulting sequence of bootstrap replications $\psi_{i}^{*}(b), m_{i}^{*}(b), \sigma_{i}^{*}(b)$ for $b=1, \ldots, B$ is recorded. The plot of a single replication of the $\log$ of $q_{x}^{*}$ 's curve and the corresponding $\log$ of $\hat{q}_{x}^{*}$ s curve, i.e., log of the fitted survival curve (5) with the parameters $\psi_{i}^{*}, m_{i}^{*}, \sigma_{i}^{*}$ is presented in Figure 2.

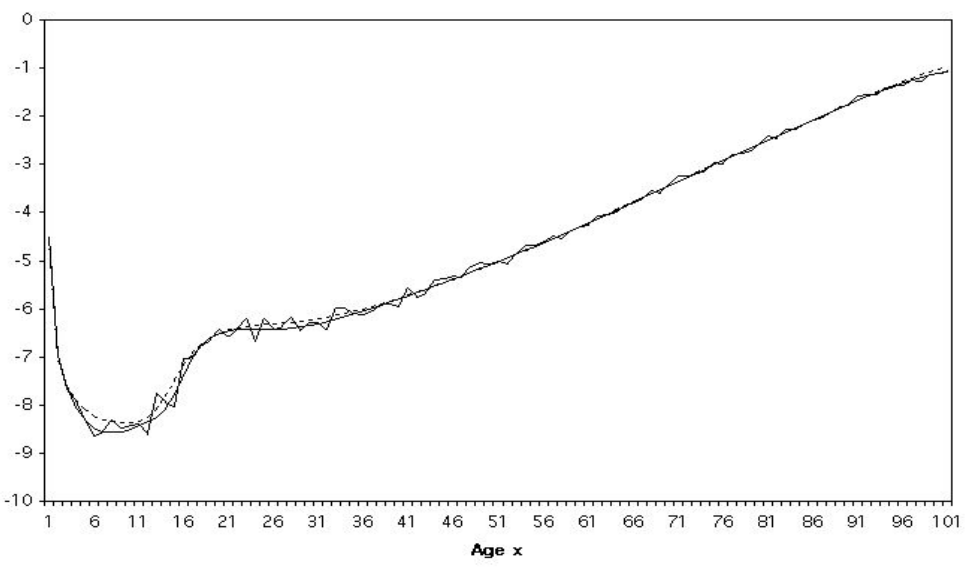

Figure 2. A plot of $\ln \left(q_{x}^{*}\right)$ (rough solid line) from a single 20,000 point resample of a parametric model (dashed line) and of $\ln \hat{q}_{x}^{*}$ (smooth solid line).

In order to find the corresponding bootstrap estimates of standard error and bias for each one of the $11 C R$ estimators in Table 1 we use the formulas (1) and (2). The corresponding bootstrap confidence interval are calculated as well, with the help of the bootstrap percentile method.

The general appropriateness of the above outlined bootstrap procedure is not immediately obvious and in fact requires some theoretical justification as it does not follow immediately from the general 
theory. Since the mathematical arguments needed to do so get quite technical and are beyond the scope of the present work, let us only briefly comment that, generally speaking, for the mere consistency of our bootstrap algorithm, the argument similar to that used to argue the consistency of $C R$ estimators may be mimicked. However, in order to show that our bootstrap estimators in fact have an edge over the normal approximation method a more refined argument based on the Edgeworth expansion theory is needed. For some insight into this and related issues see, for instance, Shao and Tu (1995) and Rempala and Szatzschneider (2002).

The final results based on our bootstrap approximations to the standard error, bias and confidence bounds for all $11 C R$ estimators given in Table 1 are presented in Table 2. As we can see from these results it seems that only the estimates of the Gompertz component ( $\left.m_{1}, \sigma_{1}, \psi_{1}\right)$ of the mortality model enjoy a reasonable degree of precision in the sense that their standard errors and biases are small (relative to the corresponding numerical value), and their confidence intervals short. For all the other component both the values for standard errors and the lengths of the confidence interval, indicate their apparent lack of precision. In particular, the lower bound of the mixture coefficient $\psi_{2}$ corresponding to the Inverse Gompertz component (6b) equals zero, which could indicate that the Inverse Gompertz component is simply not present in the considered mortality pattern. In fact, CR fitting the model (5) to US 1980 Mortality data, which has a similar pattern to the one we have considered here, did take $\psi_{2}=0$. Hence, by providing the $95 \%$ CI's for the estimates of the $\psi_{i}$ 's our bootstrap method also suggests the general form of the mixture in the Carriere law. ${ }^{3}$

For illustration purposes the distribution of $B=1000$ bootstrap

${ }^{3}$ For a formal test of significance of $\psi_{i}=0$ in a similar fashion to the usual test of the coefficients in the regression models, see, for instance, Rempala and Szatzschneider (2002). 
replications for the parameter $m_{1}$ along with its $95 \%$ confidence interval is presented in Figure 3. Let us note that the histogram of the replication values seems to approximately follow the symmetric unimodal curve which is consistent with our theoretical derivations.

As we have seen above, the parametric bootstrap algorithm can be helpful in obtaining a more complete information about the fitted mortality model and its parameters. The drawback of the algorithm presented above is mainly in its very high computational cost, since for every resample of the sequence of 11 values of the parameters we need to first obtain a resample of the fitted mortality rates $\hat{q}_{x}$, $x=1 \ldots, 100$. It would be interesting to develop some better methods of bootstrapping in the problems involving the loss functions of type (7), either with the help of the residuals bootstrap method (see Efron and Tibishirani 1993) or maybe some techniques aiming at increasing the efficient of resampling, like e.g. importance method (see Shao and Tu 1995).

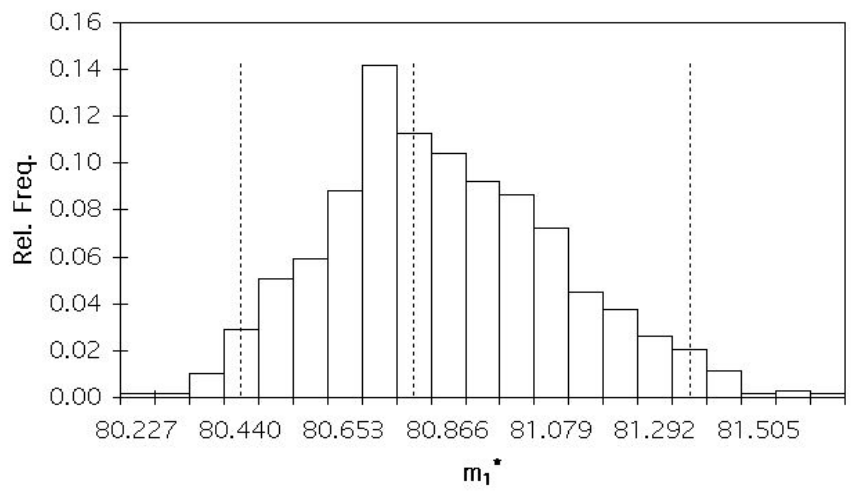

Figure 3. the distribution of $B=1000$ bootstrap replications for the parameter $m_{1}$ along with its $95 \%$ confidence interval. The vertical line in the center shown the actual fitted value of $m_{1}$ as given in Table 1. 


\begin{tabular}{l|cccccc}
\hline & $m_{1}$ & $\sigma_{1}$ & $\psi_{1}$ & $m_{2}$ & $\sigma_{2}$ & $\psi_{2}$ \\
\hline \hline Value (from Table 1) & 80.73 & 11.26 & 0.944 & 42.60 & 14.44 & 0.020 \\
SE & 0.245 & 0.278 & 0.018 & 8.257 & 4.788 & 0.015 \\
Bias & 0.096 & -0.120 & -0.003 & -1.266 & -1.653 & 0.005 \\
Lower Bd 95\% CI & 80.414 & 10.521 & 0.900 & 26.222 & 3.007 & 0.000 \\
Upper Bd 95\% CI & 81.336 & 11.608 & 0.965 & 54.531 & 19.932 & 0.056 \\
\hline \hline & $m_{3}$ & $\sigma_{3}$ & $\psi_{3}$ & $m_{4}$ & $\sigma_{4}$ & $\psi_{4}$ \\
\hline \hline Value (from Table 1) & 0.30 & 1.30 & 0.013 & 23.65 & 7.91 & 0.022 \\
SE & 0.303 & 2.268 & 0.001 & 3.011 & 3.122 & N/A \\
Bias & 0.037 & 0.254 & 0.000 & -0.179 & -0.253 & N/A \\
Lower Bd 95\% CI & 0.053 & 0.359 & 0.011 & 17.769 & 3.048 & N/A \\
Upper Bd 95\% CI & 1.290 & 10.019 & 0.015 & 30.000 & 13.504 & N/A \\
\hline
\end{tabular}

Table 2. Bootstrap estimates of the standard error, bias and $95 \% \mathrm{CI}$ for the estimators given in Table $1\left(\psi_{4}=1-\sum_{i=1}^{3} \psi_{i}\right)$.

\section{Methodology of Cash-Flow Analysis with Resampling}

In our next example we illustrate the use of nonparametric bootstrap for dependent data, by developing a whole company asset-liability model with the nonparametric model of the interest rate process. The results are compared with that based on the lognormal and stable Paretian models proposed by Klein (1993).

\subsection{Interest Rates Process}

While there exists a consensus as to the necessity of using interest rates scenarios, and to account for some form of their randomness, in the process of long-term modeling of an insurance enterprise, one can hardly argue that such a consensus is formed concerning the actual stochastic process governing the interest rates process. Becker (1991) analyzes one common model derived from the process

$$
d I_{t}=I_{t} \mu d t+I_{t} \sigma d W
$$


with $I_{t}$ denoting the interest rate prevailing at time $t, W$ denoting the standard Wiener process, with $\mu$ and $\sigma$ being the drift and volatility parameters. Becker tests the hypothesis, implied by substituting $\mu=0$ and $\sigma=$ const, that the distribution of $J_{t}=\ln \left(\frac{I_{t+1}}{I_{t}}\right)$ is normal with mean zero and constant variance. However, Becker (1991) rejects the hypothesis of normality and even independence of the $J_{t}$ 's based on the empirical evidence. Of course, (1) is not the only stochastic differential equation proposed to describe interest rate process. Many other processes have been put forth in this very active area of modern mathematical finance. Hull (1997) provides an overview of various stochastic differential equations studied in this context. De La Grandville (1998) provides a straightforward argument for approximate lognormality of the variable $(1+$ rate of return), based on the Central Limit Theorem. But Klein (1993) discusses various reasonings underlying lognormality assumption, and points out that mutual independence of consecutive returns, finite variance of one period return distribution, invariance under addition, and the functional form of the distribution, are very convenient but often unreasonable assumptions. While Klein (1993) argues convincingly against finite variance assumption, the work of Cerrito, Olson and Ostaszewski (1998), among others (notably Fama and French, 1988), aims at the evidence against independence of returns in consecutive periods. Both take away key assumptions of the Central Limit Theorem. Klein (1993) [referenced as KL in the sequel] proposes to replace lognormal distribution by the Stable Paretian, and provides a comparison of a model company under these two hypotheses. As these differing assumptions produce drastically different results, KL argues that a valuation actuary must give proper consideration to the possibility that the stochastic dynamics of the interest rate process differ significantly from the simple assumption of lognormality. In this section we propose a bootstrap-based alternative to his model as an example of the use of resampling in the nonparametric setting. 


\subsection{Modeling Interest Rates with Nonparametric Bootstrap of Dependent Data.}

Let us note that, unlike in the case of the US Mortality Tables, the historical, empirical realizations of the interest rates processes are typically available. This allows us to use the non-parametric bootstrap methods. In the following sections we investigate a non-parametric model of a company cash flow as a function of the interest rates process $\left\{J_{t}\right\}$. We do not assume independence of the $J_{t}$ 's, relaxing that assumption to $m$-dependence and stationarity of the interest rates process (see Section 3.4). We propose that it may be unreasonable to assume one functional form of the probability distribution and the choice of the non-parametric model here is not merely for the sake of example. In our opinion, this allows to proceed to deeper issues concerning the actual forms of uncertainty underlying the interest rate process, and other variables studied in dynamic financial analysis of an insurance firm. In our view, one cannot justify fitting convenient distributions to data and expect to easily survive the next significant change in the marketplace. If one cannot provide a justification for the use of a specific parametric distribution, than a nonparametric alternative should be studied, at least for the purpose of understanding firm's exposures. In this section we show the possible applicability of the resampling in studying such non-parametric alternatives.

While there are many variables involved in a company model that could be possibly investigated using our methods, in this work we shall focus exclusively on the nonparametric estimate of the distribution of 10-th year surplus value treated as a function of the underlying stochastic interest rates process. For simplicity, we assume a simple, one-parameter structure of the yield curve. Further work will be needed to extend our methodology to nonparametric estimates of the entire yield curve, as well as the liability side variables. Some recent research in this direction (Stanton 1997) provides one possible nonparametric model of term structure dynamics and the market 
price of interest rate risk. We also make a simplifying assumption of parallel shifts of all interest rates derived from the basic rate (long term Treasury rate). We follow the design of the model company in KL. That model could be generalized by extending the bootstrap techniques described here to several dependent time series (e.g., interest rate series at various maturities). Let us note that KL model assumes a parametric functional form for lapses, withdrawals, and mortality, as well as prepayments for mortgage securities in the asset portfolio. Further research into nonparametric alternatives to lapse, mortality, and prepayment models would be very beneficial to our understanding of the probability distribution structure of those phenomena. It should be noted, however, that any such research will require extensive data, which, unlike the interest rates and capital asset returns, is not always easily available.

\subsection{Model Company Assumptions}

The model company was studied effective December 30, 1990. It offered a single product: deferred annuity. The liability characteristics were as follows:

- Number of policies: 1,000

- Fund Value of Each Policy: 10,000

- Total Reserve: $10,000,000$

- Surrender Charge: None

- Minimum Interest Rate Guarantee: $4 \%$

- Reserve Method: Reserve Equals to Account Balance

The assets backing this product were: 
- 8,000,000 30-year 9.5\% GNMA mortgage pools

- 2,000,000 1 Year Treasury Bills

for a total asset balance of 10,000,000 effectively making initial surplus equal to zero.

The following initial yields in effect as of December 31, 1990 are assumed:

- 1 Year Treasury Bills: 7.00\%

- 5 Year Treasury Notes: $7.50 \%$

- 30 Year Treasury Bonds: $8.25 \%$

- Current coupon GNMAs: $9.50 \%$

Treasury yields are stated on a bond-equivalent basis, while GNMAs yields are nominal rates, compounded monthly. The following interest rates are given on 5 Year Treasury Notes at indicated dates:

- December 31, 1989: 7.75\%

- December 31, 1988: 9.09\%

- December 31, 1987: 8.45\%

- December 31, 1986: 6.67\%

The following assumptions hold about the model company:

- Lapse rate formula (based on competition and credited interest rates, expressed as percentages) is given by 


$$
q_{t}^{(w)}= \begin{cases}0.05+0.05\left[100\left(i_{\text {comp }}-i_{\text {cred }}\right)\right]^{2} & \text { if } i_{\text {comp }}-i_{\text {cred }}>0 \\ 0.05 & \text { otherwise }\end{cases}
$$

with an overall maximum of 0.5 .

- Credited interest rate $\left(i_{\text {cred }}\right)$ is a currently anticipated portfolio yield rate for the coming year on a book basis less 150 basis points. In addition to always crediting no less than the minimum interest rate guarantee, the company will always credit a minimum of $i_{\text {comp }}$ and $2 \%$.

- Competition interest rate $\left(i_{c o m p}\right)$ is the greater of 1 Year Treasury Bill nominal yield to maturity and the rate of 50 basis points below 5 year rolling average of 5 Year Treasury Bond's nominal yield to maturity.

- Expenses and Taxes are ignored.

- There is no annuitization.

- Mortgage prepayment rate $\left(\right.$ rate $\left._{t}\right)$ for the 30 year GNMA pools, calculated separately for each year's purchases of GNMAs is

$$
\text { rate }_{t}= \begin{cases}0.05+0.03\left[100\left(i_{\text {coup }, t}-i_{\text {curr }}\right)\right] & \\ +0.02\left[100\left(i_{\text {coup }, t}-i_{\text {curr }}\right)\right]^{2} & \text { if } i_{\text {comp }}-i_{\text {cred }}>0 \\ 0.05 & \text { otherwise }\end{cases}
$$

with an overall maximum of 0.40 . Here, $i_{\text {coup }, t}$ denotes the coupon on newly issued 30 year GNMAs, issued in year $t$, and $i_{c u r r}$ denotes the coupon rate on currently issued 30 year GNMAs which is assumed to shift in parallel with the yields on 30 year Treasury Bonds. We assume the same, i.e., parallel shifts, about the 1 Year Treasury Bill rates, and the 5 Year Treasury Bond rates. 
- Reinvestment strategy is as follows. If, in a given year, the net cash flow is positive, any loans are paid off first, then 1-year Treasury Bills are purchased until their book value is equal to $20 \%$ of the total book value of assets, and then newly issued current coupon GNMAs are purchased.

- Loans, if needed, can be obtained at the rate equal to current 1 Year Treasury Bills yield.

- Book Value of GNMAs is equal to the present value of the future principal and interest payments, discounted at the coupon interest rate at which they were purchased, assuming no prepayments.

- Market Value of GNMAs is equal to the present value of the future principal and interest payments, discounted at the current coupon interest rate and using the prepayment schedule based on the same rate, assuming that the current coupon rate will be in effect for all future periods as well.

- Market Value of Annuities is equal to the account value. All cash flows are assumed to occur at the end of the year. The projection period is 10 years. We run 100 scenarios. At the end of 10 years, market values of assets and liabilities are calculated. The distribution of final surplus is then compared.

\subsection{Interest Rates Process Assumptions}

In order to make a comparison of our non-parametric cash-flow analysis with the one presented in KL, we have considered the same set of yield rates on 30-years Treasury bonds from years 1977-1990, namely the average annualized yield to maturity as given in Table 2 of KL.

As indicated in KL the plot of $J_{t}$ empirical quantiles versus that of a normal random variable indicates that the $J_{t}$ 's possibly come from

a distribution other than normal. In his approach KL addressed this 
problem by considering a different, more general form of a marginal distribution for $J_{t}$, namely that of a stable Paretian family, still assuming independence of the $J_{t}$ 's. However, as it was pointed out in the discussion of his paper by Beda Chan, there is a clear statistical evidence that, in fact, the $J_{t}$ 's are not independent. The simplest argument to this end can be obtained by applying the runs test (as offered for instance by the Minitab 8.0 statistical package). There are also more sophisticated general tests for a random walk in a time series data under $\operatorname{ARMA}(p, q)$ model as offered for instance by SAS procedure ARIMA (SAS Institute 1996). The results of these tests conducted by us for the data in Table 2 of KL give strong evidence against the hypothesis of independence (both $P$-values in the above tests were less then .001).

In view of the above, it seems to be of interest to consider the validity of the stationarity assumption for $J_{t}$. Under $\operatorname{ARMA}(p, q)$ model the SAS procedure ARIMA may be used again to perform a formal test of non-stationarity, either by means of Phillips-Perron or DickeyFuller tests. However, a simpler graphical method based on a plot of an autocorrelation function (ACF) and values of the appropriate $t$-statistics (cf. e.g., Bowerman and O'Connell 1987) may also be used (if we assume that the marginal distribution of $J_{t}$ has a finite variance). In our analysis we have used both the formal testing as well as graphical methods and found no statistical evidence for nonstationarity of $J_{t}$ (all $P$-values $<.001$ ). In view of the above results, for the purpose of our non-parametric cash-flow analysis, we have made the following assumptions about the time series $J_{t}$.

(A1) The series is a (strictly) stationary time series and

(A2) has the $m$-dependence structure, i.e., $t$-th and $s+t+m$-th elements in the series are independent for $s, t=1,2, \ldots$ and some fixed integer $m \geq 1$.

The assumption (A1) implies that all the $J_{t}$ 's have the same marginal 
distribution but with no conditions regarding the existence of any of its moments. Thus a random variable $J_{t}$ could possibly have an infinite variance or even infinite expectation (which is the case for some distributions from the stable Paretian family). As far as the assumption (A2) is concerned, the ACF plot in Figure 2 indicates that in the case of long-term Treasury Bonds from years 1953-1976, an appropriate value of $m$ is at least 50. The assumption of $m$-dependence for $J_{t}$ seems to be somewhat arbitrary. In fact, from a theoretical viewpoint the condition (A2) may be indeed substantially weakened by imposing some much more general but also much more complicated technical conditions on the dependence structure of the $J_{t}$ 's. These conditions, in essence, require only that $J_{t}$ and $J_{t+s}$ are "almost" independent as $s$ increases to infinity at appropriate rate and are known in the literature as mixing conditions. Several types of mixing conditions under which our method of analysis is valid are given by Shao and Tue (1995 p. 410) For the sake of simplicity and clarity of a presentation, however, we have not considered these technical issues here.

\subsection{Bootstrapping the Surplus-Value Empirical Process}

In the sequel, let $M$ denote a 10-th year surplus value of our company described in Section 3.3. In general, as indicated above $M$ is a complicated function of a variety of parameters, some deterministic and some stochastic in nature, as well as the investment strategies. However, since in this work we are concerned with modeling cash flow as a function of underlying interest rates process, for our purpose we consider $M$ to be a function of the time series $\left\{J_{t}\right\}$ satisfying assumptions (A1) and (A2) above, assuming all other parameters to be fixed as described in Section 3.3. Under these conditions, we may consider $M$ to be a random variable with some unknown probability distribution $H(x)=\operatorname{Prob}(M \leq x)$. Our goal is then to obtain an

estimate of $H(x)$. To this end, we consider a set of $n=167$ values 
of $J_{t}$ process obtained from annualized 30-year Treasury bond yield rates as given in Table 2 of KL. If we make an assumption that for the next 10 years the future values of increments of $\ln I_{t}$ will follow the same stationary process $J_{t}$, we may then consider calculating the values of $M$ based on these available 167 data values. In fact, since the J's in Table 2 of KL are based on the monthly rates, every sequence of 120 consecutive values of that series gives us a distinct value of $M$ which results in obtaining $n-120+1=46$ empirical values of $M$. However, by adopting the "circular series" idea, i.e., by wrapping the 167 values from Table 1 around the circle and then evaluating $M$ for all sequences of 120 "circular consecutive" $J_{t}$, we obtain additional 119 empirical values of $M$. Thus a total of $n=167$ empirical values of $M$ is available. The cumulated distribution of these values is plotted in Figure 4 as the thick curve. It is important to note that the obtained empirical $M$ 's are not independent but rather form a time series which inherits the properties (A1) and (A2) of the original time series $J_{t} \cdot{ }^{4}$ A natural approximation for $H(x)$ is now an empirical process $\widehat{H}_{n}(x)$ based on the values, say, $m_{1}, \ldots m_{n}$ of $M$ obtained above $(n=167)$. That is, we put

$$
\widehat{H}_{n}(x)=\frac{1}{n} \sum_{i=1}^{n} I\left(m_{i} \leq x\right)
$$

where $I\left(m_{i} \leq x\right)=1$ when $m_{i} \leq x$ and 0 otherwise. By a standard result from the probability theory, under the assumptions that the $m_{i}$ 's are realizations for a time series satisfying (A1) and (A2) we have that for any $x$

$$
\widehat{H}_{n}(x) \rightarrow H(x) \quad \text { as } n \rightarrow \infty .
$$

\footnotetext{
${ }^{4}$ Note that this statement is not entirely correct here, as there is, perhaps, a small contamination due to the use of the "wrapping" procedure for calculating empirical values of $M$. However, it can be shown that the effect of the circular wrapping quickly disappears as the number of available $J_{t}^{\prime} s$ grows large. For the purpose of our analysis we have assumed the wrapping effect to be negligible.
} 
As we can see from the above considerations, the empirical process $\widehat{H}(\cdot)$ could be used to approximate the distribution of the random variable $M$ and, in fact, a construction of such an estimator would typically be a first step in any non-parametric analysis of this type. In our case, however, the estimate based on the empirical values of the $m_{i}$ 's suffers a drawback, namely that it uses only the available empirical values of the surplus value, which in turn rely on the very long realizations of the time series $J_{t}$ while in fact explicitly using only very few of them. Indeed, for each $m_{i}$ we need to have 120 consecutive values of $J_{t}$, but in the actual calculation of the surplus value we use only 10 . This deficiency of $\widehat{H}_{n}(\cdot)$ may be especially apparent for small and moderate values of the dependence constant $m$ in (A2), i.e., when we deal only with a relatively short dependence structure of the underlying $J_{t}$ process (and this seems to be the case, for instance, with our 30 year Treasury yields data).

In view of the above, it seems reasonable to consider a bootstrapped version of the empirical process (9), say $\widehat{H}_{n}^{*}$, treated as a function of the underlying process $J_{t}$ with some appropriately chosen block length $l$. Thus, our bootstrap estimator of $H(x)$ at any fixed point $x$ (i.e., $\hat{\theta}_{n}^{*}$ in our notation of Section 1.4, with $\widehat{H}_{n}$ being $\hat{\theta}_{n}$ ) is

$$
\widehat{H}_{n}^{*}(x)=\frac{1}{B} \sum_{b=1}^{B} \widehat{H}_{(b)}^{*}(x)
$$

where $\widehat{H}_{(b)}^{*}(x)$ is a value of $\widehat{H}(x)$ calculated for each particular realization of the pseudo-values $J_{1}^{*}, \ldots, J_{n}^{*}$ generated via a circular bootstrap procedure using $k$ blocks of length $l$ as described in Section 1.4, and $B$ is the number of generated pseudo-sequences. In our case we have taken $B=3000$. The first four realizations of the process $\widehat{H}_{n}^{*}(x)$ are presented in Figure $4{ }^{5}$ Let us note that the above es-

\footnotetext{
${ }^{5}$ In order to achieve a desired accuracy of the bootstrap estimator for $m$-dependent data,the number of generated resamples is typically taken to be fairly large (typ-
} 


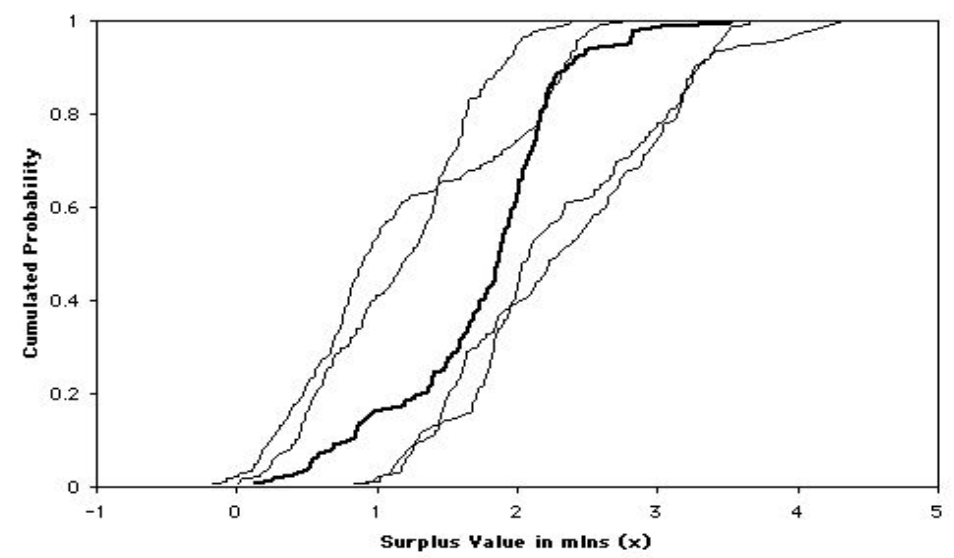

Figure 4. Empirical surplus value process $\widehat{H}(x)$ (thick line) and the four realizations of the bootstrapped surplus value process $\widehat{H}_{n}^{*}(x)$.

timate may be also viewed as a function of the bootstrapped surplus values of $M^{*}$, say, $m_{1}^{*}, \ldots, m_{n}^{*}$ obtained by evaluating the surplus value for pseudo-values of the series $J_{1}^{*}, \ldots, J_{n}^{*}$. For the purpose of the interval estimation of $H(x)$, we consider bootstrap confidence intervals based on the method of percentiles, as described in Section 1.3 where now the bootstrap distribution $G_{*}$ denotes the distribution of $\widehat{H}_{n}^{*}(x)$ at any fixed point $x$, given the observed values of $J_{1}, \ldots, J_{n}$.

In the next two sections, using the cash flow model outlined in Section 3.3, we analyze the distribution of the 10-th year surplus using the data for average annualized yields to maturity for 30 years Treasury Bonds. For the sake of comparison we also present the results of a similar analysis based on a larger set of data, namely on the average yield to maturity on long-term Treasury Bonds for the period 1953-1976 as given in Table 4 of KL.

ically, at least 1000). Of course, the number of all possible distinct resamples is limited by the number of available distinct series values $(n)$ and a block length $(l)$. 


\subsection{Bootstrap Estimates of the Surplus Cumulative Distribution}

The bootstrap estimates $\widehat{H}_{n}^{*}(\cdot)$ of the 10-th year surplus values distribution $H(x)=\operatorname{Prob}(M \leq x)$ for a grid of $x$ values ranging between -3 and 4 (millions of dollars) along with their $95 \%$ confidence intervals were calculated using a method of circular bootstrap as described in Section 1.4 with a circular block length of six months (i.e., in notation of Section $1.4, l=6$ ). Other values of $l$ were also considered and the obtained results were more or less comparable for $1<l<6$. In contrast, the results obtained for $l \geq 7$ were quite different. However, the latter were deemed to be unreliable in view of a relatively small sample size $(n=167)$, since the theoretical result on the block bootstrap states that $l$ should be smaller then $\sqrt{n}$ in order to guarantee the consistency of the bootstrap estimates (cf. Shao and Tu, 1995 Chapter 9). The case $l=1$ corresponds to an assumption of independence among the $J_{t}$ 's and was not considered here.

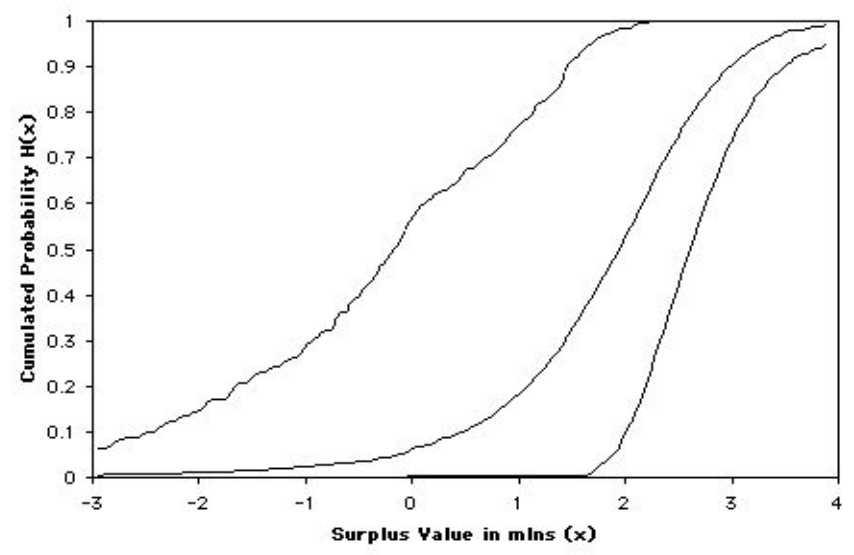

Figure 5. The plot of $\widehat{H}_{n}^{*}(x)$ along with its $95 \%$ confidence bounds.

The plot of the bootstrap estimate of $H(x)=\operatorname{Prob}(M \leq x)$ given by (10), along with the $95 \%$ confidence bounds is presented in Figure 5 . In order to achieve a reasonable accuracy the number of gener- 
ated resamples for each calculated point $x$ was taken to be $B=3000$. The values of the estimators, together with their $95 \%$ confidence intervals for some of the values between -2.5 and 4 (in millions of dollars), are also presented below in Table 3 .

\begin{tabular}{ccc}
\hline $\begin{array}{c}\text { Surplus } \\
\text { Value } x\end{array}$ & $\begin{array}{c}\text { Bootstrap } \\
\text { Estimator } \widehat{H}_{n}^{*}(x)\end{array}$ & $95 \%$ CI \\
\hline \hline-2.50 & 0.01 & $(0.00,0.10)$ \\
-1.75 & 0.01 & $(0.00,0.17)$ \\
-1.00 & 0.02 & $(0.00,0.29)$ \\
-0.50 & 0.04 & $(0.00,0.40)$ \\
0.00 & 0.06 & $(0.01,0.57)$ \\
0.50 & 0.10 & $(0.01,0.67)$ \\
1.00 & 0.18 & $(0.01,0.77)$ \\
1.75 & 0.42 & $(0.02,0.96)$ \\
2.00 & 0.53 & $(0.10,0.98)$ \\
2.50 & 0.75 & $(0.42,1.00)$ \\
3.00 & 0.91 & $(0.74,1.00)$ \\
3.50 & 0.97 & $(0.90,1.00)$ \\
4.00 & 1.00 & $(0.96,1.00)$ \\
\hline
\end{tabular}

Table 3: Bootstrap estimates of $H(x)$ for selected values of $x$ along with their $95 \%$ confidence bounds.

Let us note that often for merely practical reasons we would be more interested in estimating $1-H(x)$, i.e., a probability that surplus value will exceed certain threshold, rather then $H(x)$ itself. However, these estimates can be easily obtained from Table 3 as well. For instance, in order to obtain an estimate of a positive 10-th year surplus $(\operatorname{Prob}(M>0))$ we subtract the estimate of $\mathrm{H}(0)$ given in Table 3 from one. The resulting estimate is then $1-\widehat{H}_{n}^{*}(0)=1-0.06=$ 0.94. In similar fashion the upper and lower confidence bounds for $1-H(0)$ can be obtained as well. The estimates of probability of exceeding of several different threshold values $x$ are given in Table 4 . From the values reported in either table it is easy to see that our bootstrap estimates based on average yields on 30 years Treasury Bonds 
1977-1990 are not precise for $x$ values in the interval from -2 to 2 million, in the sense that they have very wide confidence intervals. For instance, the estimated probability of a negative 10-th year surplus equals 0.06 but its interval estimate with $95 \%$ confidence is in fact a value between 0.01 and 0.57 . Hence, in reality, the true value of this probability could be as high as 0.57 . The graph of a bootstrap estimate of the 10-th year surplus distribution $H(x)=\operatorname{Prob}(M \leq x)$, along with its $95 \%$ confidence bounds, based on the values of the $J_{t}$ 's calculated from average yields on 30 years Treasury Bonds 19771990 is given in Figure. The values of $\widehat{H}_{n}^{*}(x)$ were calculated for a very dense grid of $x$ values in order to give an appearance of a continuous curve.It seems, however, that this lack of precision was not the bootstrap's fault, but rather an effect of a relatively small sample size for the observed values of the time series. In order to verify whether or not this was truly the case we have conducted our bootstrap analysis using also a different set of data, namely the data on the average yields of the long term Treasury bonds from the period 1953-76 as given in Table 4 of KL.

\begin{tabular}{ccc}
\hline$x$ & $1-\widehat{H}_{n}^{*}(x)$ & $95 \%$ CI \\
\hline \hline 0 & 0.94 & $(0.43,0.99)$ \\
1 & 0.82 & $(0.23,0.99)$ \\
2 & 0.47 & $(0.02,0.90)$ \\
3 & 0.09 & $(0.00,0.26)$ \\
\hline
\end{tabular}

Table 4. Bootstrap estimates of $1-H(x)$ along with their confidence bounds obtained from the estimates given in Table 3 .

\subsection{Estimates Based on the Yields on the Long-Term Treasury Bonds for 1953-76}

Applying the same methodology as above we have re-analyzed the Klein company model using the data for the average yield to maturity on the long-term Treasury bonds for years 1953-1976. For selected values of $x$ the numerical values of the bootstrap estimates of $H(x)$ 


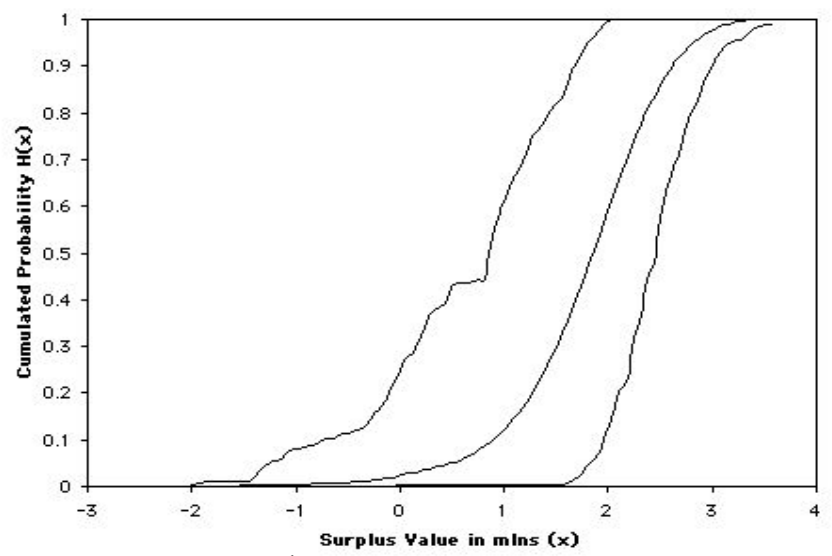

Figure 6. The plot of $\widehat{H}_{n}^{*}(\cdot)$, along with its $95 \%$ confidence bounds using the longterm Treasury bonds data for years 1953-1976.

based on this data set are presented in Table 5. The plot of the $\widehat{H}_{n}^{*}(x)$ calculated for sufficiently many $x$ values so as to give it an appearance of a continuous curve, along with its $95 \%$ confidence bounds, is presented in Figure 6. Let us note that in this case the bootstrap estimate is indeed more precise (in the sense of generally shorter confidence intervals) then the one presented in Figure 5 and based on the 30-years Treasury Bond data for 1977-1990, especially in the range between -1 and 1 . In particular, the probability of the negative 10 -th year surplus is seen to be estimated as 0.03 with $95 \%$ confidence interval between 0 and 0.24 . Hence, it seems that using the time series data on the interest rates spanning over 24 years (as opposed to 14 years for 30-year Treasury) we were able to make our bootstrap estimates more precise, especially in the middle part of the 10-th year surplus distribution. It would be useful to further investigate that phenomenon by considering even longer periods of time and study the effect of the long time series data sets on the accuracy of our bootstrap estimators. Of course a danger of considering too long data sets is that over a very long period of time the key assumptions (A1) and (A2) of Section 3.4 may no longer be valid. 


\begin{tabular}{ccc}
\hline $\begin{array}{c}\text { Surplus } \\
\text { in mlns }(x)\end{array}$ & $\begin{array}{c}\text { Bootstrap } \\
\text { Estimator } \widehat{H}_{n}^{*}(x)\end{array}$ & $95 \%$ CI \\
\hline \hline-1.75 & 0.00 & $(0.00,0.01)$ \\
-1.00 & 0.00 & $(0.00,0.08)$ \\
-0.50 & 0.01 & $(0.00,0.11)$ \\
0.00 & 0.03 & $(0.00,0.24)$ \\
0.50 & 0.05 & $(0.00,0.43)$ \\
1.00 & 0.12 & $(0.00,0.61)$ \\
1.75 & 0.44 & $(0.03,0.93)$ \\
2.00 & 0.59 & $(0.12,1.00)$ \\
2.50 & 0.86 & $(0.57,1.00)$ \\
3.00 & 0.98 & $(0.90,1.00)$ \\
3.50 & 1.00 & $(0.99,1.00)$ \\
\hline
\end{tabular}

Table 5: Bootstrap estimates of $H(x)$ along with their $95 \%$ confidence bounds for the longterm Treasury bonds data for 1953-1976.

\subsection{Comparison with the Parametric Analysis Results}

In KL an analysis of the 10-th year surplus for a company model described in Section 3.3 was presented under the assumptions that (1) the random variables $J_{t}$ are independent and identically distributed, and (2) that they follow some prescribed probability distribution. In his paper KL has considered two competing parametric classes: lognormal and stable Paretian. By estimating the parameters in both models with the help of the data for average yield to maturity on 30-years Treasury Bonds for years 1977-1990 and then generating a large number of interest rates scenarios, via Monte Carlo simulations (the techniques similar to parametric bootstrap described in Section 2), KL has arrived at the empirical estimates of the distributions for the 10-th year surpluses under two different models.

KL pointed out that the probability of negative surplus under a stable Paretian model seems to be much higher then under a lognormal one. In Figure 7 we present the empirical distributions of the 10th year surplus based on 100 random scenarios as reported in KL, 
along with our bootstrap estimate. Based on the naive comparison of the three curves it seems that our bootstrap estimate resembles more closely the distribution obtained by KL under the lognormal interest rates model, and in particular, does not indicate the probability of a negative surplus to be as high as indicated under the stable Paretian model for the underlying process of interest rates. However, keeping in mind the confidence bounds on our bootstrap estimate presented in Figure 5, one would have to be very cautious about making such conclusions. In fact, since KL in his method of approximating the true distribution of the 10-th year surplus did not consider any measure of error of his estimators, a simple comparison of the point values on the three curves presented in Figure 7 may be quite misleading. It would be certainly more beneficial for the sake of the validity of our comparisons if an error estimate was produced for the surplus distributions of KL, for instance via the methods described in Chapter 2.

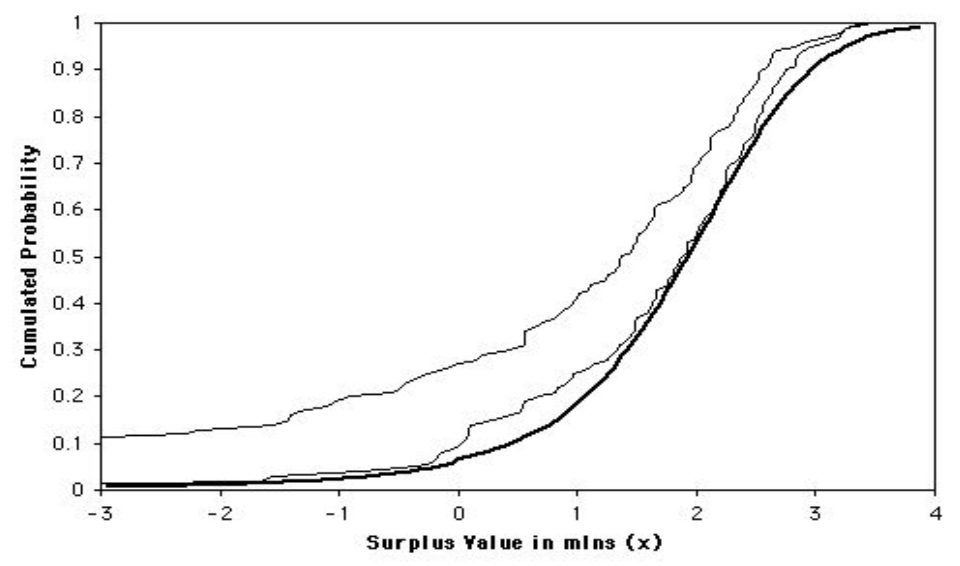

Figure 7. The plot of $\widehat{H}_{n}^{*}(\cdot)$ from Figure 5 (lowest curve) along with the empirical c.d.f.'s obtained by KL. Upper curve corresponds to a stable Paretian and the middle one to the lognormal model. 


\section{Conclusions}

The assessment of the fitted model variability is one of the most important aspects of statistical modeling. In this work we have shown that the bootstrap, in both the parametric and the non-parametric setting, can be quite useful in accomplishing this goal for the actuarial models. In mortality modeling this allows for the better understanding of the fitted model and its sensitivity to the changes in the underlying mortality patterns. The benefits of our approach are even more apparent in cash flow testing model since the stochastic structure there is even richer, and probably still less understood.

The remarkable feature of the bootstrap method applied to the interest rates process was that under quite minimal assumptions we were able to obtain the model for cash flow testing which seems to perform equally well, if not better then the parametric ones. The models of this work are, hopefully, the first step towards the development of new actuarial methodologies in modeling of insurance enterprises. Some additional inroads into modeling of loss distributions, inferences about the severity of loss, loss distributions percentiles and other related quantities based on data smoothing, as well as bootstrap estimates of standard error and bootstrap confidence intervals for losses have been given, for instance, by Derrig, Ostaszewski and Rempala (2000). We believe that this area holds a great promise for dynamic future research which we would like to promote and encourage with this work.

\section{Acknowledgments}

The authors wish to acknowledge Ms Kim Holland for her expert help in typsetting this document. 


\section{References}

Actuarial Standards Board (1991), "Performing Cash Flow Testing for Insurers," Actuarial Standard of Practice No. 7 Washington, D.C.

Becker, D. N. (1991), "Statistical Tests of the Lognormal Distribution as a Basis for Interest Rate Changes," Transactions of the Society of Actuaries. 43, 7-72.

Bickel, P. J. and Freedman, D. A. (1981), "Some asymptotic theory for the bootstrap," Ann. Statist. 9, no. 6, 1196-1217.

Black, F. and Myron S. (1973), "The Pricing of Options and Corporate Liabilities," Journal of Political Economy. 81 No. 3, 637-654.

Bowerman, B. and O’Connell, T. (1987), Time Series Forecasting. Duxbury Press, Boston.

Cerrito, P., Dennis O., and Ostaszewski, K. (1998), "Nonparametric statistical tests for the random walk in stock prices," Advances in Quantitative Analysis of Finance and Accounting. 6, 27-36.

de La Grandville, O. (1998), "The Long-Term Expected Rate of Return: Setting It Right," Financial Analysts Journal. NovemberDecember, $75-80$.

Derrig, R., Ostaszewski, K., and Rempala, G. (2000), "Applications of resampling methods in actuarial practice," Proceedings of the Casualty Actuarial Society, 87, 322-364.

Efron, B. (1979), "Bootstrap methods. Another look at Jackknife," Ann. Statist. 7, no. 1, 1- 26. 
"Efron, B. and Tibshirani, R. (1993)," An Introduction to the Bootstrap. Chapman and Hall, New York.

Fama, E. and French, K. (1988), "Permanent and Temporary Components of Stock Market Returns," Journal of Political Economy. 96, 246-273.

Hsu, D., Miller, R., and Wichern, D. (1974), "On the Stable Paretian Behavior of Stock-Market Prices," Journal of the American Statistical Association 69, 108-113.

Hull, J. (1997), Options, Futures, and Other Derivative Securities, 3rd ed., Prentice-Hall Simon Schuster.

Klein, G. (1993), "The Sensitivity of Cash-Flow Analysis to the Choice of Statistical Model for Interest Rate Changes," Transactions of the Society of Actuaries. 45, 9-124. Discussions 125-186.

Künsch, H. (1989), "The jackknife and the bootstrap for general stationary observations," Ann. Statist. 17, no. 3, 1217-1241.

Ostaszewski, K. (2002) Asset-Liability Integration, Society of Actuaries Monograph M-F102-1, Actuarial Education and Research Fund, Schaumburg, Illinois, 2002.

Panjer, H. (editor) (1998) Financial Economic with Applications to Investments, Insurance and Pensions, Actuarial Education and Research Fund, Schaumburg, Illinois, 1998.

Politis, D. and Romano, J. (1992), "A circular block-resampling procedure for stationary data", in Exploring the limits of bootstrap, (R. LaPage, editor) 263-270, Wiley, New York.

Rempala, G. and Szatzschneider, K. (2002), "Bootstrapping Para- 
metric Models of Mortality", manuscript, to appear in Scandinavian Actuarial Journal.

Ross, S. (1976), "The Arbitrage Theory of Capital Asset Pricing," Journal of Economic Theory. 13, 341-360.

SAS 8.2 Statistical Package (2001), SAS Institute, Cary NC.

Shao, J. and Tu, D. (1995), The jackknife and bootstrap, SpringerVerlag, New York.

Sharpe, W. F. (1964), "Capital Asset Prices: A Theory of Market Equilibrium under Conditions of Risk", Journal of Finance. 19, $425-442$.

Singh, K. (1981), "On the asymptotic accuracy of Efron's bootstrap", Ann. Statist. 9, no. 6, 1187-1195.

Stanton, R. (1997), "A Nonparametric Model of Term Structure Dynamics and the Market Price of Interest Rate Risk", The Journal of Finance. 52 No. 5, 1973-2002.

Tilley, J. A. (1992), “An Actuarial Layman's Guide to Building Stochastic Interest Rate Generators", Transactions of the Society of Actuaries. 44, 509-538. Discussions 539-564.

Vanderhoof, I. T. and Altman, E., editors (1998), Fair Value of Insurance Liabilities. Kluwer Academic Publishers. 